\title{
Carrier-Envelope Phase Stabilization of Grating Based High-Power Ultrafast Laser
}

\author{
Shouyuan Chen, Yi Wu, Kun Zhao and Zenghu Chang \\ Kansas State University, Manhattan, KS 66503
}

USA

\section{Introduction}

At a fixed point of the space, the electric field of a Fourier transform-limited laser pulse can be written as:

$$
E(t)=A(t) \exp \left(i \omega_{0} t+\varphi_{C E}\right),
$$

where $A(t)$ is the pulse amplitude, which is peaked at $t=0$, and $\omega_{0}$ is the laser pulse central frequency. Carrier-envelope (CE) phase, $\varphi_{C E}$, denotes the offset between the peak of the pulse envelope and the nearest peak of the carrier-wave electric field as shown in Fig. 1.

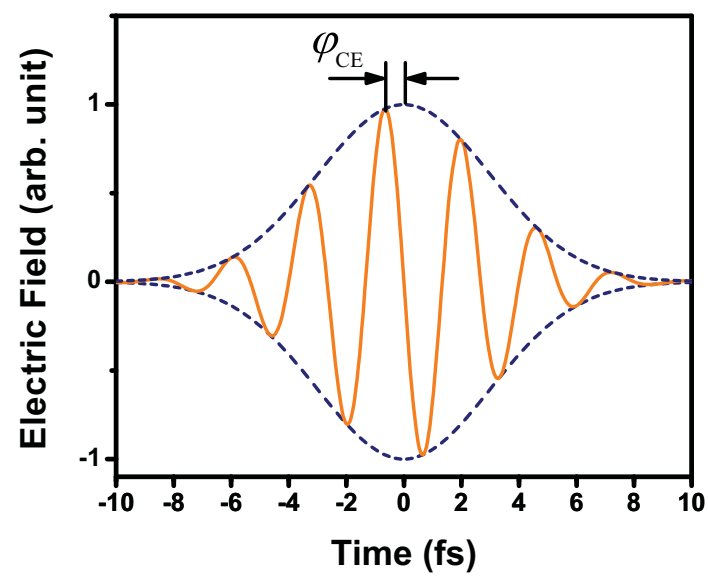

Fig. 1. A few-cycle laser pulse showing the carrier-envelope offset, $\varphi_{C E}$.

When the width of $A(t)$ approaches few or single-cycle duration, CE phase starts to play an important role in the laser-matter interactions becuase the electric-field amplitude changes rapidly within half of the cycle depending on the different CE phase. This fast variation of the electric field within the pulse envelope is the origin of CE phase effects in a variety of high-field processes, such as above-threshold ionization, high-order harmonic generation and molecular dissociation (Paulus et al., 2001; Haworth et al., 2007; Kling et al., 2006). The importance of $\mathrm{CE}$ phase are even identified in terahertz emission spectroscopy with few- 
cycle pulses (Kreb et al., 2006). Advances in CE phase control also make it possible to controlling such processes as injected photocurrents in semiconductors (Fortier et al., 2004) and in sub-single- cycle pulse trains generated with Raman sidebands (Chen et al., 2008). One of the important applications of the CE phase stabilization is the isolated attosecond generation (Hentschel et al., 2001; Sansone et al., 2006; Mashiko et al., 2008; Feng et al., 2009). For generating attosecond pulses using double optical gating (DOG) or general double optical gating (GDOG), the effective electric field inside the gate can be expressed as

$$
E(t)=g(t) \cos \left(\omega_{0} t+\varphi_{C E}\right),
$$

where $g(t)$ is the gating function with a duration of a fraction of a laser cycle and the center of the gate occurs at $t=0$. In these cases, the opening time of the gate for single attosecond pulse extraction is of the order of half to one cycle (Sansone et al., 2006; Mashiko et al., 2008). Thus, it is crucial to stabilize and control the CE phase. In Section 3 of this paper, the effects of $\mathrm{CE}$ phase on polarization gating and double optical gating will be explored in detail.

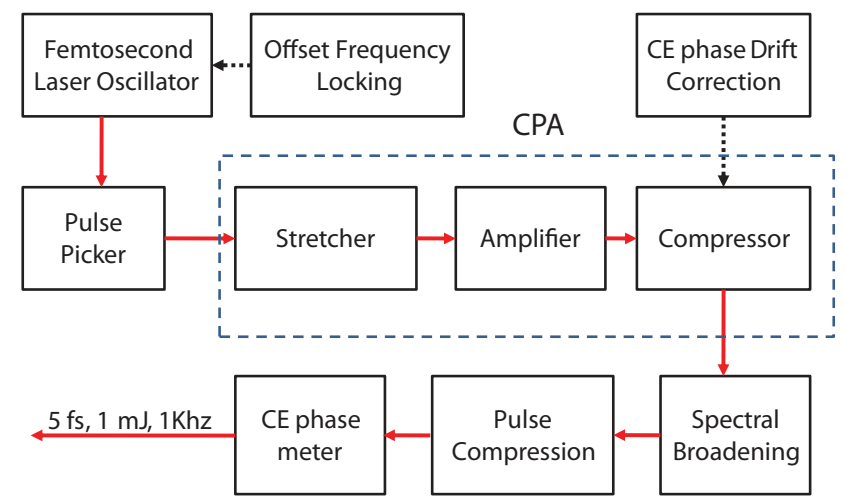

Fig. 2. A schematic diagram of a CE phase stable laser system producing few-cycle pulses.

A typical laser system for generating few-cycle CE phase-stabilized pulses is shown in Fig. 2. First, the CE phase evolution in the oscillator is stabilized and the pulses with the same CE phase are selected and sent to the chirped pulse amplification (CPA) system, where the laser pulses are temporally stretched, amplified, and recompressed (Strickland \& Mourou (1985)). The CE phase drift introduced by the amplifier stage is also corrected. The amplified laser pulses are then spectrally broadened through a nonlinear process and compressed to only a few cycles in duration. Finally, the CE phase of the few-cycle pulses is measured and stabilized.

The CE phase stabilization of the laser pulse generated from an amplifier with dispersive material based stretcher and compressor had been successfully demonstrated before (Baltuška et al., 2003). However, the low damage threshold of the dispersive material limits the laser pulse energy to about $1 \mathrm{~mJ}$, which ultimately limits the flux of the attosecond pulse generated from such laser system. It is of much current interest to increase the attosecond pulse flux for many attosecond experiments and extend the attosecond physics to nonlinear regime (Schultze et al., 2010; Tzallas et al., 2003). On the other hand, the grating based amplifier has been successfully demonstrated to generate PW class ultrafast pulse (Kiriyama et al., 2010), which can be used for high flux attosecond pulse generation, and thus it is 
important to study the CE phase evolution in such laser system and stabilize the CE phase of the laser pulse of the grating based amplifier.

\section{CE phase stabilization by controlling the grating separation}

\subsection{CE phase shift caused by grating shift in stretcher and compressor}

For the grating based stretcher and compressor, it has been shown that the pointing stability of the laser beam on the gratings may introduce CE phase noise to the amplified femtosecond pulses (Kakehata et al., 2002; Thomann et al., 2004). It was soon shown that the grating separation could also cause the CE phase drift of amplified laser pulses (Chang, 2006).

To understand the CE phase drift caused by the stretcher and compressor, we start with the analysis of the laser pulse phase variation after it propagate through a stretcher. A typical setup of the stretcher is shown in Figure 3. The analysis is also valid for grating compressors.

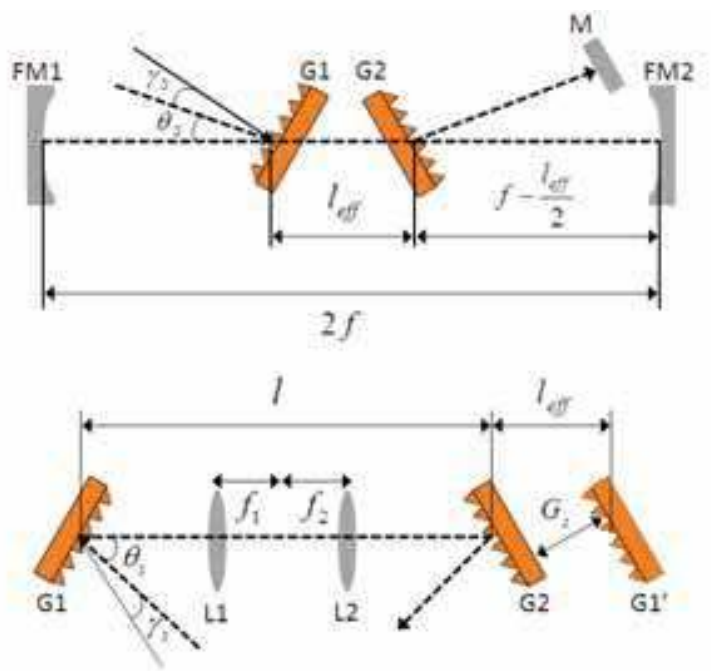

Fig. 3. Grating stretcher. The top figure uses mirrors to form the telescope and the bottom figure uses lenses to form the telescope. In both figures: G1 and G2 are the gratings, FM1 and FM2 are the focusing mirrors, $\gamma_{s}$ : the incidence angle on the first grating, $\theta_{s}$ : the angle between the diffracted beam and incident beam, $l_{e f f}$ : the effective grating separation, G1: the image of G1 in the bottom figure (Moon et al., 2010).

If we assume the pulses to be transform limited at the stretcher, in time domain, the pulses can be expresses as Eq. 1. In spectral domain, the electric filed is

$$
E(\omega)=E_{0}(\omega) \exp \left[i\left(\varphi_{C E}+\phi(\omega)\right)\right]
$$

where $\left|E_{0}(\omega)\right|$ is the power spectrum and $\phi(\omega)$ is the spectral phase, which is also equal to zero. After the pulse propagates through the double-pass grating compressor, the CE phase shift was:

$$
\Delta \varphi_{C E}=\omega_{0} \tau\left(\omega_{0}\right)-\varphi^{\prime}\left(\omega_{0}\right)=4 \pi\left(\frac{G_{S}}{d_{s}} \tan \left[\gamma_{s}-\theta_{s}(\omega)\right]\right)
$$


Where $\omega_{0} \tau\left(\omega_{0}\right)$ is the group delay, $\phi^{\prime}\left(\omega_{0}\right)$ is the phase delay, $G_{s}$ is the effective perpendicular distance between gratings, $d_{s}$ is the grating constant, $\gamma_{s}$ is the angle of incidence, and $\theta_{s}(\omega)$ is the diffraction angle. From the geometry,

$$
G_{s}=-l_{\text {eff }} \cos \left(\gamma_{s}-\theta_{s}\right),
$$

where $l_{\text {eff }}$ is the effective linear distance between the gratings, the CE phase shift becomes:

$$
\Delta \varphi_{C E}=-4 \pi \frac{\Delta l_{\text {eff }}}{d_{s}} \sin \left(\gamma_{s}-\theta_{s}\right)
$$

Considering the incident angle is close to the Littrow angle, and the grating constant is of the order of wavelength (grating Constance $d=1200 \mathrm{~g} / \mathrm{mm}$ is commonly used), the CE phase approximates:

$$
\frac{\Delta \varphi_{C} E}{\Delta l_{e f f}}=2 \pi \frac{\Delta l_{e f f}}{d_{s}^{2}} \approx \frac{2 \pi}{\lambda} .
$$

Thus, Eq. 7 shows that a change in the grating separation comparable to a wavelength will yield a CE phase change of $2 \pi$. Eqs. 5 and 7 also realized how the CE phase can be controlled and stabilized by changing the grating separation. $\lambda$

\subsection{CE phase stabilization of multi-pass amplifier}

The experimental setup used in determining the effect of the grating separation on the CE phase is carried on Kansas Light Source (KLS) laser system, which contains a multi-pass amplifier, as shown in Fig. 4. One of the mirrors in the stretcher was placed in a piezoelectronic transducer (PZT) controlled mount. CE-phase stable pulses from the oscillator were sent to the CPA system. An f-to-2f interferometer (Kakehata et al., 2002), spectrometer, and computer measured the spectral interferometry signal from which the CE phase was extracted. In the f-to-2f, the laser was focused into a sapphire plate for spectral broadening and then the infrared components were frequency doubled by a BBO crystal. Finally, a polarizer was used to select a common polarization and the beam was sent to a spectrometer. The resulting interferogram was analyzed by a computer to retrieve the CEphase drift. For each data point, 50 laser shots were integrated. In the experiment, a 60-V sinusoidal voltage was applied to the PZT and observed. As a comparison, a DC voltage was applied to the PZT. The results are shown in Fig. 5 (Li, Moon \& Chang, 2006). It was determined from the measurement that a $1 \mu \mathrm{m}$ change in grating separation introduced a 3.7 $\pm 1.2 \mathrm{~S}$ rad phase shift, which is consistent with the calculation of Eq. 7 .

The grating separation in the stretcher was then used as a feedback control to stabilize the CE-phase drift of the amplified pulses. This is shown in Fig. 6, where the CE-phase error was kept to $160 \mathrm{mrad}$ rms over $800 \mathrm{~s}$. 50 laser shots were integrated for each data point. This was the standard experimental procedure for all CE-phase measurements in the KLS laboratory. The bottom plot shows the PZT movement during the same period. The concept of controlling the stabilized CE phase is illustrated in Fig. 7 (Li et al., 2006). In Fig. 7, the grating separation was precisely controlled to scan the phase over a range of $2 \pi$. In the experiment, the setpoint for locking the phase was changed from $1.1 \pi$ to $0.9 \pi$ in steps of $0.2 \pi$. 


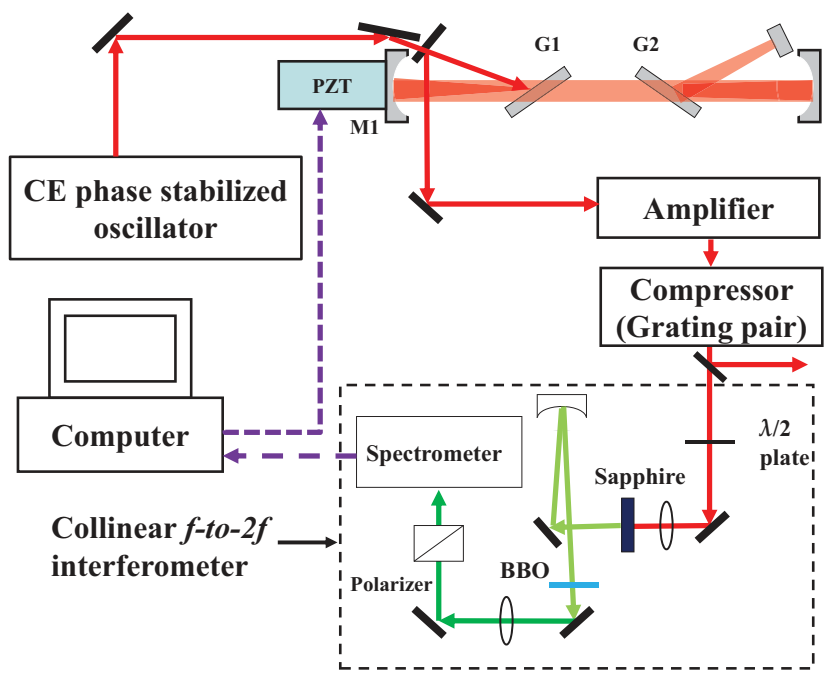

Fig. 4. Kansas Light Source laser system for testing the effects of the grating separation of the stretcher on the CE-phase stability. G1 and G2 are the gratings. M1 is one of the telescope mirrors driven by a piezoelectronic transducer (PZT) (Moon et al., 2010).
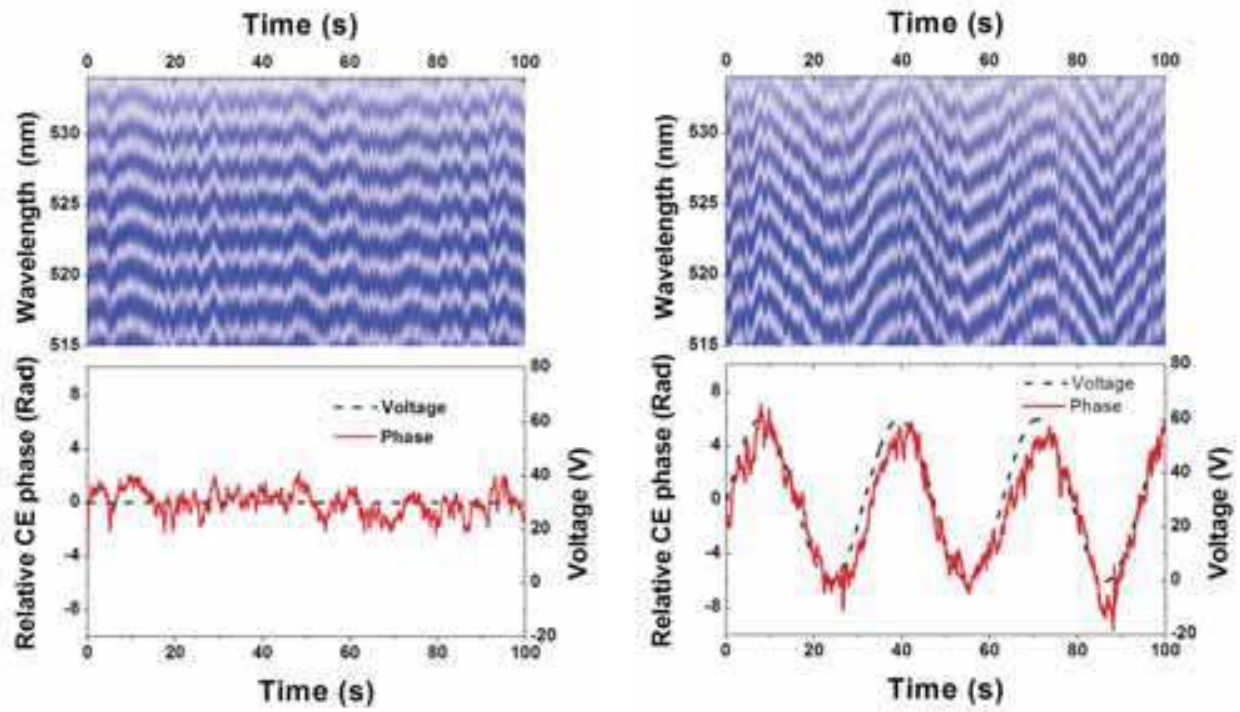

Fig. 5. Dependence of the CE phase of the amplified pulses on the grating separation. (a) Fringe pattern and (b) corresponding relative $C E$ phase obtained with a $30-\mathrm{V}$ DC voltage applied to the PZT. (c) Fringe pattern and (d) corresponding relative CE phase obtained with a $60-\mathrm{V}$ sinusoidal voltage applied to the PZT, which caused the PZT to move $3.6 \mu \mathrm{m}$ (Li, Moon \& Chang, 2006). 


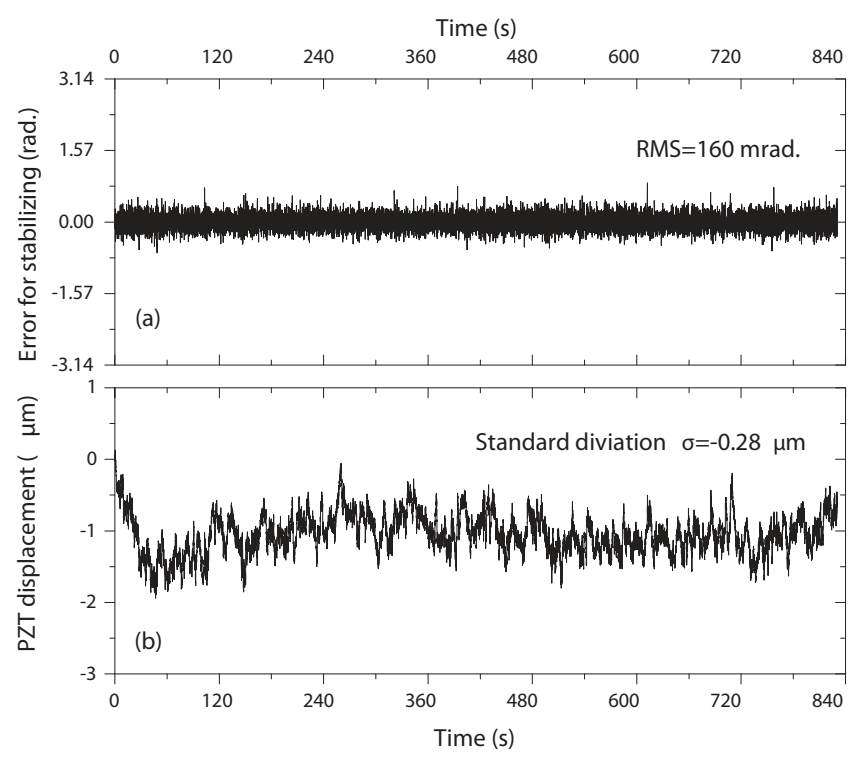

Fig. 6. (a) The error signal for the slow feedback stabilization, (b) the displacement of PZT when the set-point was shifted (Li et al., 2006).

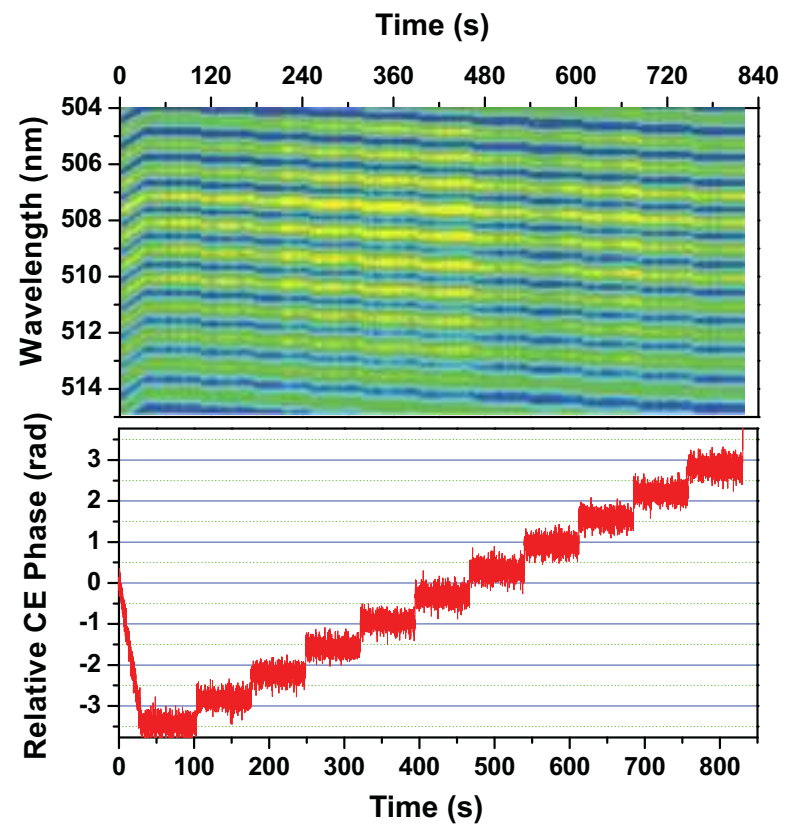

Fig. 7. Precisely controlling the CE phase in amplified pulses. Top: the temporal evolution of interference fringes measured with a collinear $f-2 f$ interferometer. Bottom: the swept $\mathrm{CE}$ phase (Li et al., 2006). 
The relative CE phase was kept at each setpoint for 1 minute and then moved to the next setpoint. During the process, the phase error was smoothly locked to an average of $161 \mathrm{mrad}$ rms, which showed how well the gratings could stabilize the $\mathrm{CE}$ phase and change the $\mathrm{CE}$ phase simultaneously. Note that in the algorithm used, the first detected fringe pattern was phase zero. Therefore, the CE phase was changed relative to the first detected phase, which had an unknown value.

Similarly, the grating separation in a compressor can also be used to control and stabilize the CE phase. The size of the optic to be used as a control mechanism should be considered when choosing to use either the stretcher or compressor. A large optic, such as a large mirror in the stretcher, would be harder to move using a PZT than a smaller optic. Also, a larger bandwidth of CE-phase noise could be suppressed with a smaller optic. The effects of controlling the grating separation in a compressor arrangement were investigated ( $\mathrm{Li}$ et al., 2008). The experimental setup used to investigate the compressor control is shown in Fig. 8. Controlling the compressor grating separation was found to stabilize the CE phase to 230 mrad rms over $270 \mathrm{~s}$, which was nearly the same as the stretcher grating performance. Fig. 9 shows the performance of the compressor grating separation control. The top plot shows the difference between the situation where the feedback control was turned on and when it was inactive. The bottom plot shows the fast Fourier transform of the phase drift. The plot shows how the feedback control corrects CE-phase error under $4 \mathrm{~Hz}$. This is expected since the drift of CE phase-stable pulses through an amplifier is slow, since the oscillator stabilization corrects the majority of the fast drift. However, as in (Moon et al., 2006), locking the pathlength difference in the oscillator CE-phase stabilization interferometer will reduce the fast noise (>3 Hz) by over $40 \%$, which can improve the overall CE-phase-locking quality of the system.

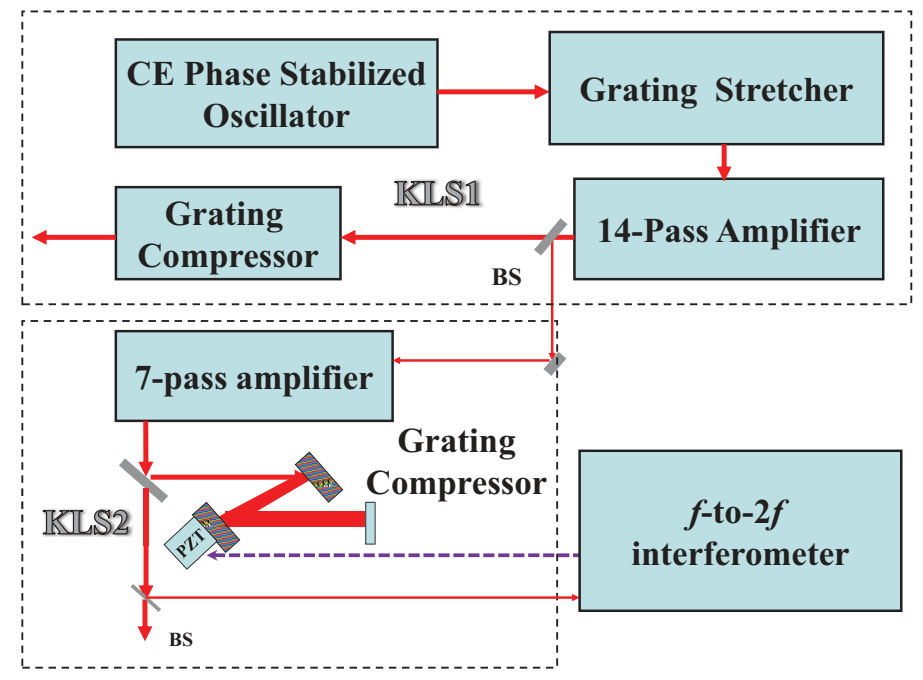

Fig. 8. Experimental setup for controlling the CE phase of the amplified laser pulses using the compressor grating ( $\mathrm{Li}$ et al., 2008). BS: beamsplitter. 

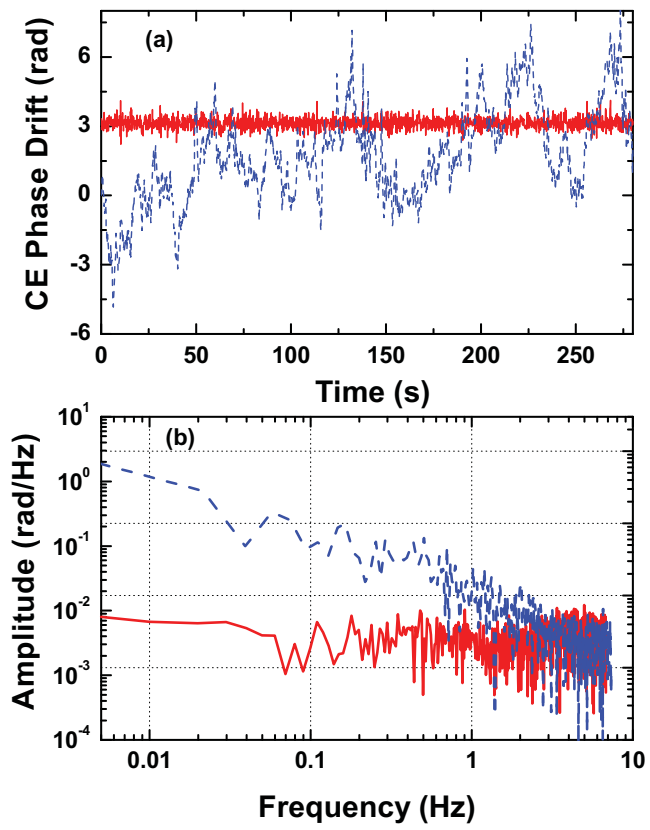

Fig. 9. (a) The evolution of the freely drifting (dotted line) and stabilized (solid line) CE phase. (b) The fast Fourier transform of the CE-phase drift under the free-running (dotted line) and stabilized conditions (solid line) (Li et al., 2008).

\subsection{CE stabilization of regenerative amplifier}

Besides the multi-pass amplifier, regenerative amplification is another attractive choice for generating ultrafast laser pulses with multi-mJ pulse energy at high repetition rates (Vaillancourt et al., 1990; Barty et al., 1996). Compared with multi-pass amplifiers, the laser pulses generated from regenerative amplification have better beam profile, pointing stability, power stability and extraction efficiency. It has been commonly used as the preamplifier for high energy femtosecond laser systems. However, the CE phase stabilization has been demonstrated previously only on multi-pass lasers (Baltuška et al., 2003; Li et al., 2008). Although the CE phase evolution after the regenerative amplifier has been explored to some extent (Kakehata et al., 2004), the CE phase drift caused by the amplification stage has not been corrected. Furthermore, no high-field CE phase dependent experiments have been demonstrated with a regenerative amplifier. We demonstrate the $\mathrm{CE}$ phase stabilization and control of the Manhattan Attosecond Radiation Source (MARS) regenerative amplification laser system and unambiguously confirm the stability by measurement of the $\mathrm{CE}$ phase dependence of the high order harmonic spectrum generated by double optical gating (Chen et al., 2009; Mashiko et al., 2008).

The MARS laser system consists of a commercially available Ti:Sapphire Coherent Legend Elite Duo ${ }^{\mathrm{TM}}$ chirped pulse amplifier $(\mathrm{CPA})$ operating at $1 \mathrm{KHz}$, seeded by a Femtolasers Rainbow ${ }^{\mathrm{TM}}$ oscillator. The oscillator operates at $78 \mathrm{MHz}$ repetition rate with a $200 \mathrm{mWoutput}$ power. The carrier-envelope offset frequency $\left(f_{C E O}\right)$ of the oscillator is stabilized using the 


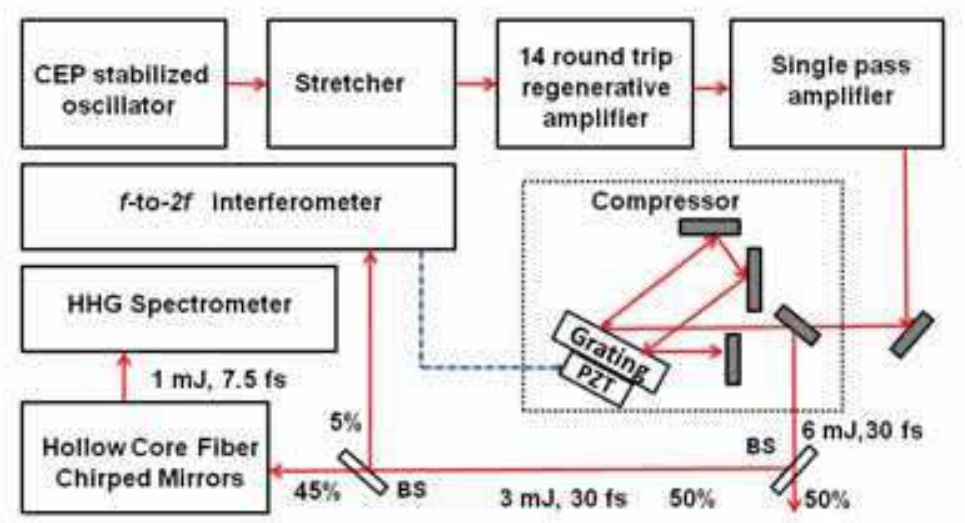

Fig. 10. Layout of the MARS laser system Chen et al. (2009). BS: beam splitter.

monolithic carrier-envelope phase-stabilization scheme (Fuji et al., 2005). With the assistance of the temperature feedback control, $f_{C E O}$ of this oscillator can be locked about 12 hours on a daily basis (Yun et al., 2009). As shown in Figure 10, the amplifier consists of a grating based stretcher, two amplification stages and a grating based compressor.

The stretcher stretches the pulse to about 160 ps. The first amplification stage is a 14 round trip regenerative amplifier, which amplifies the laser pulse to $4 \mathrm{~mJ}$. The second stage is a single pass amplifier to boost the laser pulse energy to $8 \mathrm{~mJ}$. The Ti:Sapphire crystals in both amplification stages are thermoelectricity cooled to $-12{ }^{\circ} \mathrm{C}$. The regenerative amplifier and single pass amplification stages are each pumped by $50 \%$ of a 45 WCoherent Evolution HETM pump laser. After compression, the final output pulse energy is $6 \mathrm{~mJ}$ with a central wavelength of $800 \mathrm{~nm}$ and spectral bandwidth of $37 \mathrm{~nm}$, which supports a fourier transform limited pulse duration of $28 \mathrm{fs}$. The pulse duration as measured with FROG is $30 \mathrm{fs}$. The CE phase drift of the amplified pulse is measured by sending partial of the output beam to the f-to-2f interferometer (Kakehata et al., 2001). The feedback signal is then sent to a piezoelectric transducer (PZT) stage on the compressor grating to stabilize CE phase slow drift by controlling the grating separations (Li et al., 2008; Chang, 2006). Because of the high output power of the MARS laser system, the laser beam is typically split by a 50/50 beam splitter to support two experiments simultaneously. During the CE phase stabilization process, it was found that $\mathrm{CE}$ phase after this amplifier system is very sensitive to mechanical vibration and acoustic noise. One reason for this sensitivity is that in Regen cavity, all the laser passes follow the exact same beam path. Any perturbation of air in the cavity will be amplified by the pass number. It is also the major difference between the Regen and Multi-pass amplifier. In the later configuration, the laser beam follow different pathes, and thus has less sensitivity than Regen on the acoustic noise. However, by moving the pump laser further away from the amplifier, padding the laser cover with sound absorption materials and improving the stability of the optical mounts in the stretcher and compressor, the CE phase stability was achieved.

The RMS error of the locked CE phase was measured to be $90 \mathrm{mrad}$ over a period of 4.5 hours with $50 \mathrm{~ms}$ spectrometer integration time as shown in Fig. 11. The locking duration is sufficient for many low count rate experiments, such as attosecond streaking and laser ion 
beam interaction experiments, which can require several hours of CE phase stabilization (Wang et al., 2009; McKenna et al., 2008). To control the CE phase in the experiment, the grating separation of the compressor was stabilized at preset values. As shown in Fig. 12, the CE phase was swept from $-\pi$ to $\pi$ for many cycles, which can be used to observe the CE phase effects in high harmonic and attosecond pulse generation experiments (Li et al., 2006). Some regions in the Fig. 11a and Fig. 12a show transient reductions of the fringe visibility, which are likely due to acoustic noise and vibration in the laboratory which could not be avoided over such a long locking period. However, such small disturbances did not affect the quality of the CE phase control.

To unambiguously confirm the CE phase stabilization of MARS laser system, the dependence of the high order harmonic spectrum generated in Argon gas from DOG was measured using an XUV transmission grating spectrometer (Shan \& Chang, 2001). To generate the short pulse ( $<10 \mathrm{fs}$ ) required by DOG, half of the laser pulse with an energy of 3 $\mathrm{mJ}$ was sent to a $1 \mathrm{~m}$ long hollow-core fiber with an inner diameter of $400 \mathrm{~m}$ and filled with

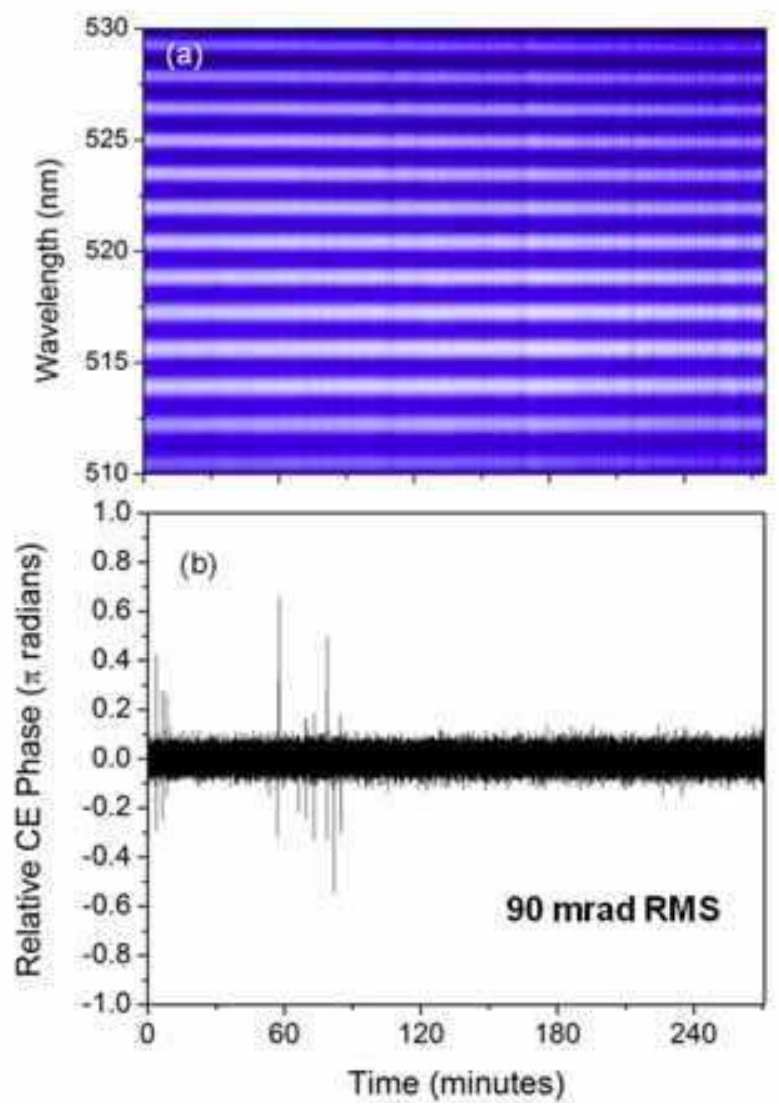

Fig. 11. Long term CE phase stabilization. (a) f-to-2f interference fringes. (b) Retrieved CE phase from the fringes, which has 90 mrad RMS averaged over 50 laser shots Chen et al. (2009). 


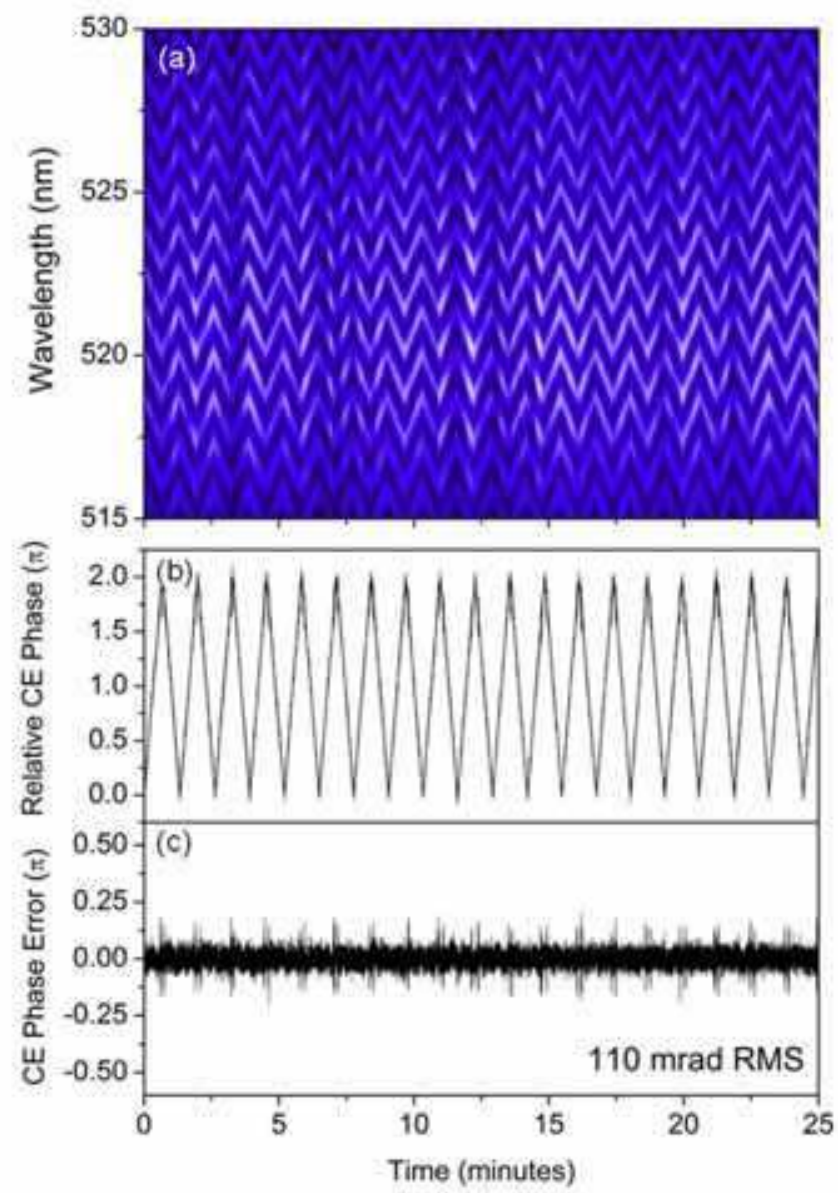

Fig. 12. Periodic CE Phase sweep from $-\pi$ to $\pi$. (a) f-to-2f interferometer fringes, (b) The scanning phase value retrieved from the fringes, (c) CE Phase error of retrieved phase relative to the preset scanning phase. The periodic increases in error are due to the PZT response at the turning points of control curve Chen et al. (2009).

Neon gas at a pressure of $2 \mathrm{~atm}$. After self-phase modulation, the laser pulse spectrum was broadened from $600 \mathrm{~nm}$ to $950 \mathrm{~nm}$. The Frequency-Resolved-Optical-Gating measurement shows the pulse duration is $7.5 \mathrm{fs}$ after it was compressed by the chirped mirrors, which is short enough for generating the single isolated attosecond pulse (Gilbertson et al., 2008). The final output power of the short pulse is $1 \mathrm{~mJ}$ after the chirped mirrors. The short pulse was then focused by a $300 \mathrm{~mm}$ spherical mirror into a $1.5 \mathrm{~mm}$ gas cell filled with Argon at 40 torr to generate the high order harmonics. When the CE phase was scanned linearly from 0 to $8 \pi$, the harmonic spectra varied with a $2 \pi$ periodicity as shown in Fig. 13, which is consistent with the $2 \pi$ periodicity of the electric field generated by DOG (Chang, 2007). The total time to perform the CE phase scan was 30 minutes. 


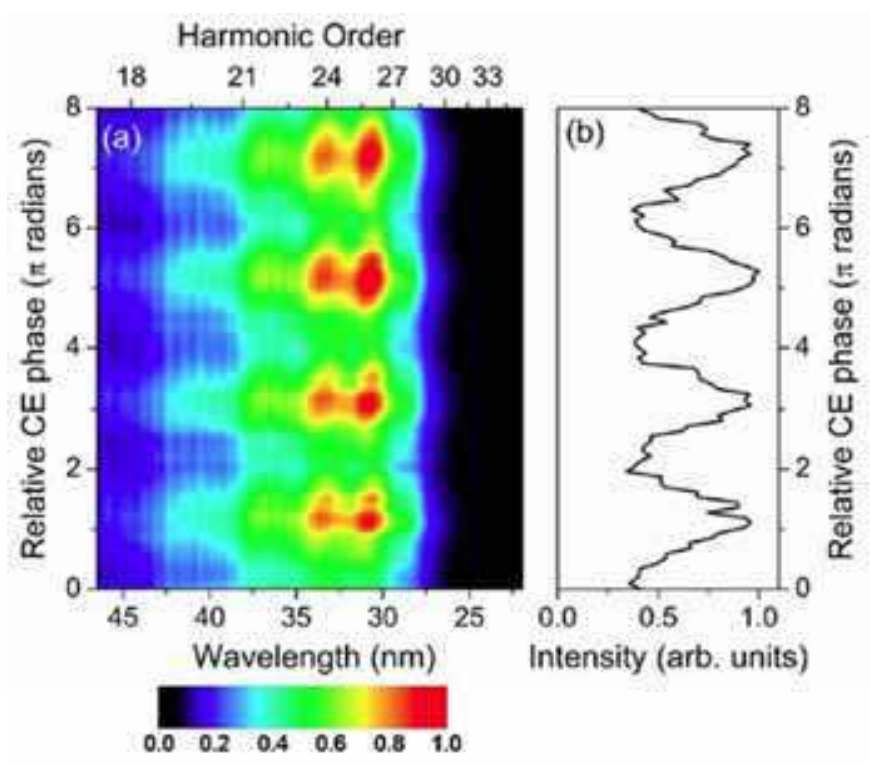

Fig. 13. (a) DOG harmonic spectra taken with the CE phase scanned from $0 \pi$ to $8 \pi$. (b) Line out of the normalized integrated spectrum. The integration range is from $48 \mathrm{~nm}$ to $20 \mathrm{~nm}$. The $2 \pi$ periodicity is consistent with the asymmetric electric field of DOG Chen et al. (2009).

\section{Isolated attosecond pulse generation with CE phase stabilized high-power laser}

As mentioned in the previous section, one of the important applications of CEP stabilized laser is to generate isolated attosecond pulses.

Attosecond pulse generation is usually interpreted in the semi-classical re-collision model (three-step model) (Corkum, 1993; Corkum \& Chang, 2008). Briefly, as a strong near infrared (NIR) laser pulse strikes an atom, a free electron wave packet is produced by ionization. Once freed, the wave packet moves away from the atom. However, when the oscillating laser electric field reverses direction, half of the packet is driven back towards the parent ion. This return electron can recombine with the parent ion, emitting an extreme ultraviolet (XUV) photon, which is the origin of attosecond XUV pulses. In general, a multi-cycle laser will produce an attosecond XUV pulse every half of a laser cycle. The result is a train of attosecond pulses. It is obvious that the CEP is critical in the isolated attosecond pulse generation with a gating technique. It is preferred that the CEP of the NIR laser pulse is stabilized so that the center of the gate always overlaps with a single attosecond XUV pulse in the pulse train. If the CEP is not optimized, the pulse energy of the single attosecond pulse would be reduced or, in the worst scenario, multiple attosecond pulses will be generated instead of an isolated attosecond pulse.

To study the relation between the CE phase and attosecond pulse generation, the isolated attosecond pulse generation and characterization experiments were performed in the KLS lab (Feng et al., 2009; Gilbertson et al., 2010). 

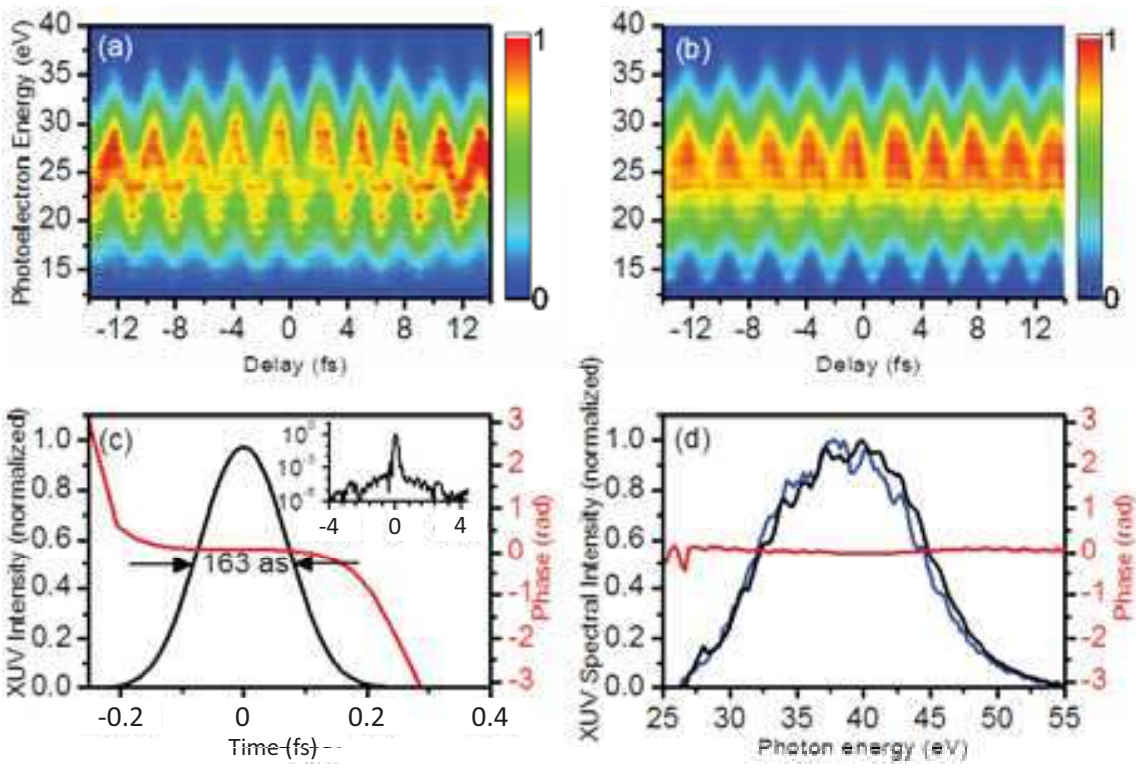

Fig. 14. The experimentally obtained (a) and retrieved (b) spectrograms of isolated attosecond pulses streaked by multicycle laser pulses. The temporal profile (solid line) and phase (dotted line) are shown in (c). The inset figure shows the same temporal profile but over an extended range. The pre- and post-pulses located at \pm 2600 as are less than $0.1 \%$ of the main pulse. Panel (d) shows the experimental (dashed line) and retrieved (solid line) XUV-only spectrum. The dashed-dotted line shows the spectral phase and indicates that the pulse is nearly transform limited (Gilbertson et al., 2010).

Figure 14 shows the results of the temporal characterization of isolated attosecond pulses produced by GDOG technique using a streak camera setup (Feng et al., 2009; Gilbertson et al., 2010) and the frequency resolved optical gating for the complete reconstruction of attosecond bursts (FROG-CRAB) method (Mairesse \& Quéré, 2005). Figures 14(a) and (b) show the experimental and reconstructed streaked spectrograms, respectively. Figure 14(c) shows the temporal profile of the pulse (solid line) and the temporal phase (dotted line). The full width at half maximum (FWHM) of the pulse is about 163 as. The inset figure shows the temporal profile over an extended range, which indicates the contributions from pre- and post pulses are less than $0.1 \%$ of the main peak. This shows that the pulse is indeed an isolated attosecond pulse. Figure 14(d) shows a comparison between the experimental XUVonly spectrum (dashed line) and the retrieved spectrum (solid line) from the retrieved temporal profile and phase shown in Fig. 14(c). This marginal check indicated the reconstructed results can be trusted and the pulse is nearly transform-limited.

The gate width of the GDOG in the above experiment was set equal to one optical cycle, or roughly 2.5 fs. This is the upper limit for generating isolated attosecond pulses with a proper $\mathrm{CE}$ phase. The gate width can be further reduced so that it is much less than one optical cycle. Figures 15(a) and (b) show the electric field of the driving laser with two values of the $C E$ phase within the gate. The color gradient indicates the ellipticity of the generating laser pulse with white being the most linear. Here, the gate width was chosen to 


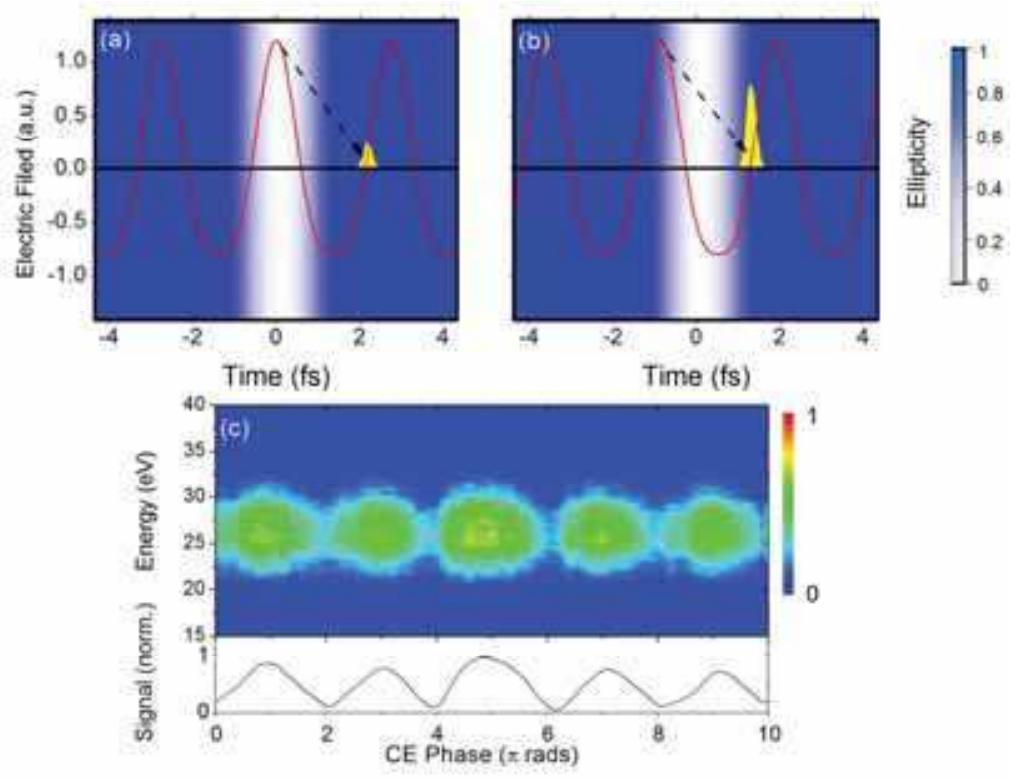

Fig. 15. The effect of a narrow gate width $(\sim 1 \mathrm{fs})$ on the generated attosecond pulse. In (a), the CE phase of the NIR laser forces the freed electron recombines in a field of high ellipticity, severely limiting its recombination probability. In (b), the CE phase is more favorable for highflux attosecond pulse emission since the electron experiences a linear field for its full lifetime. In the figures, the color gradient represents the ellipticity of the field with blue being the most elliptical and white the most linear. The experimental evidence for this effect is shown in (c). The upper figure shows the energy spectrum as a function of the CE phase of the NIR laser while the lower plot shows the total signal integrated along the energy axis. The $2 \pi$ periodic structure is the effects of the two-color gating in GDOG (Gilbertson et al., 2010).

be $\sim 1$ fs (about half of a laser cycle) and is where the attosecond pulse is produced. In Fig. $15(\mathrm{a})$, the freed electron is born during a strongly linearly polarized portion but recombines to emit an XUV photon in a field that is increasingly elliptical. This reduces the recombination probability so that the attosecond XUV photon flux would be low. In Fig. 15(b), the electron spends all of its excursion time away from the parent ion in a mainly linearly polarized field so that the attosecond photon flux would be maximized. In both cases, since the gate width is much smaller than the spacing between two adjacent attosecond pulses in the pulse train, it is not possible to generate two attosecond pulses per laser shot. The CE phase only affects the flux of the isolated pulses.

Figure 15(c) shows the experimental evidence for this effect. For this portion of the experiment, a $9 \mathrm{fs}$ laser pulse was produced by the $2 \mathrm{~mJ}, 25 \mathrm{fs}$ NIR pulse from the CEPlocked amplifier passing through a $\mathrm{Ne}$ filled hollow-core fiber and a chirp-mirror compressor. The laser power fluctuates less than $1 \%$. This beam then passed through the GDOG optics consisting of a $530 \mu \mathrm{m}$ quartz plate, a $0.5 \mathrm{~mm}$ Brewster window, a $440 \mu \mathrm{m}$ quartz plate and a $141 \mu \mathrm{m} \mathrm{BBO}$, and was focused by an $\mathrm{f}=375 \mathrm{~mm}$ spherical mirror into a 1.4 $\mathrm{mm}$ long Ar gas target. The gate width for these parameters was calculated to be about $1.4 \mathrm{fs}$. 
The upper figure in Fig. 15(c) shows the energy spectrum of the photoelectrons liberated by an attosecond XUV pulse as a function of the CE phase of the input NIR laser. The CE phase was continuously shifted from 0 to $2 \pi$. Typically, the CE phase stability is better than 250 mrads after the hollow-core fiber (Mashiko et al., 2007). Two features of the spectrogram are obvious. First, the spectrum is a continuum for all CE phase values, which satisfies the necessary condition for generating isolated attosecond pulses. Second, the intensity of the spectrum strongly depends on the CE phase, which is expected for such a narrow gate width. The lower figure shows the total counts (integrated over the energy spectrum) as a function of the CE phase. The modulation depth is an indication of the width of the linear polarization gate. For narrower gate widths, the modulation depth would become even stronger while for wider gate widths, the modulation would become shallower and eventually the energy spectrum would exhibit modulations indicative of multiple pulses within the gate (Sola et al., 2006).

The attosecond XUV pulses generated under different CEP values are also characterized by the attosecond streak camera. A streaked spectrogram similar to the one shown in Fig. 14 was obtained when the CE phase is unlocked. The carrier of the laser field is not smeared out since the attosecond pulse is automatically locked to the driving laser oscillation in time. The temporal profile and phase as reconstructed by FROG-CRAB are also similar to the ones in Fig. 14. The pulse duration was found to be about 182 as.

Then, streaked spectrograms for four different values of the CE phase of the input laser were taken, as Figure 16 shows. The CE phase was locked to a $200 \mathrm{mrad}$ RMS. The differences in count rates are attributed to the different values of the CE phase and hence the different fluxes of the attosecond XUV photons. Figure 17(a) shows the XUV spectrum at each
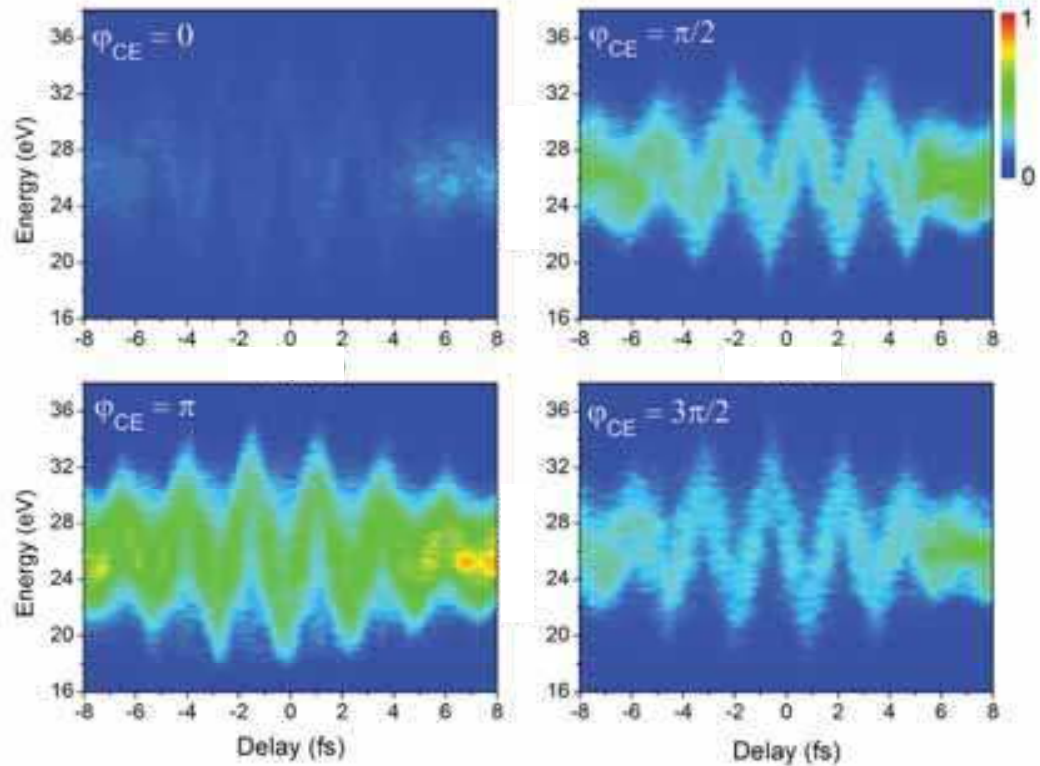

Fig. 16. Streaked photoelectron spectrograms for four different values of the CE phase, $\sim 0 \mathrm{rad}, \sim \pi / 2 \mathrm{rad}, \sim \pi \mathrm{rad}$, and $\sim 3 \pi / 2 \mathrm{rad}$. The images are normalized to the peak counts of the $\sim \pi \mathrm{rad}$ spectrogram Gilbertson et al., (2010). 
value of the CE phase. The temporal profiles and phases for the spectrograms in Fig. 16 were reconstructed with FROG-CRAB (Mairesse \& Quéré, 2005) and all the pulse durations are about 180 as. Finally, each streaked spectrogram was Fourier filtered to extract the oscillating NIR field. Figure 17(b) shows the results, where the CE phase of the 9 fs laser pulse can be easily seen.

To improve the utility of this result, attosecond pulses were produced using $25 \mathrm{fs}$ NIR pulses directly from the chirped pulse amplifier. Figure 18 shows streaked spectrograms for two different values of the $\mathrm{CE}$ phase. Again, the count rate is different between the two cases in agreement with the attosecond pulse dependence on the CE phase. Reconstructions with FROG-CRAB show both have nearly identical durations of 190 as and phase shapes. The signal ratio between the two cases is not as extreme as the short pulse case. This can

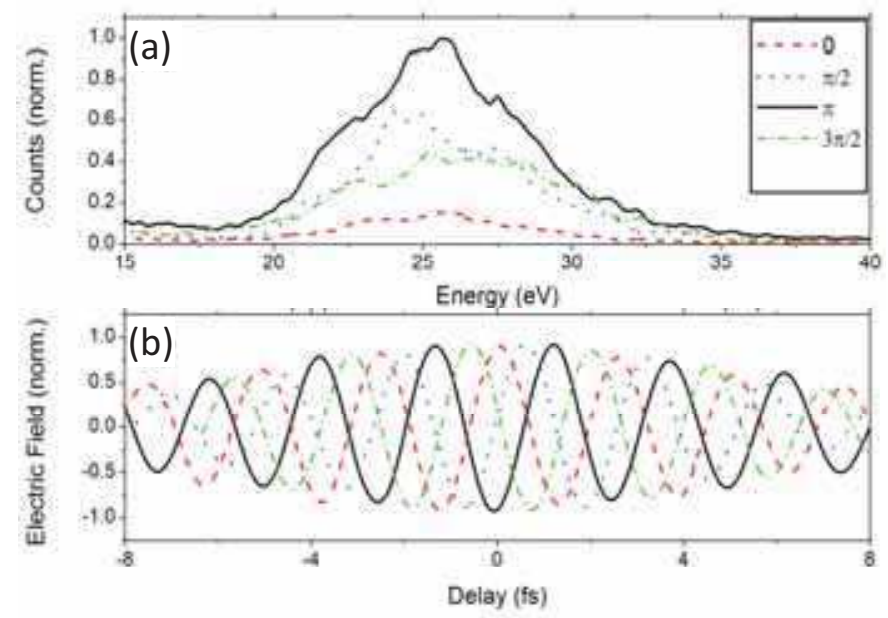

Fig. 17. Panel (a) shows the photoelectron energy spectrum for each of the streaked spectrograms in Fig. 16. Panel (b) shows the extracted NIR laser electric fields corresponding to each of the spectrograms in Fig. 16 Gilbertson et al., (2010).
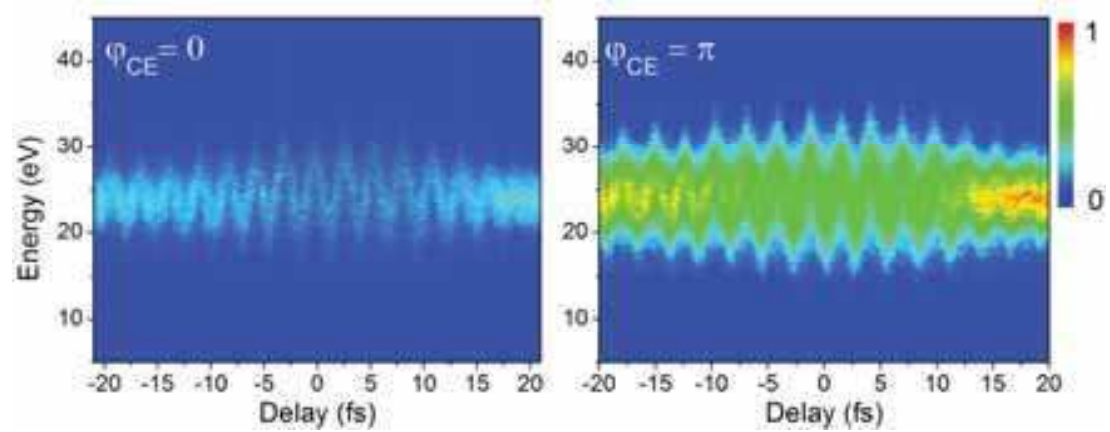

Fig. 18. Streaked spectrograms of attosecond pulses produced directly from an amplifier with an approximately $\pi$ CEP shift between them Gilbertson et al., (2010). 
possibly be explained by the gate width being slightly wider than the short pulse case. This is in excellent agreement with the CE phase unlocked reconstruction of 190 as.

These results show that the CEP locking plays a key role in single attosecond XUV pulse generation with a gating technique, DOG or GDOG (Feng et al., 2009; Gilbertson, Wu et al., 2010; Gilbertson, Khan et al., 2010). Although the single attosecond pulses produced under different CEP have almost identical pulse duration and phase profile, the photoelectron count rate or the flux of the XUV photos in the isolated attosecond pulses varies significantly as the CEP changes. As we extend the HHG spectrum to higher energy range to generate even shorter XUV pulses, 25 as, for example, which is about one atomic unit of time (Mashiko et al., 2009), the efficiencies of both XUV photon emission in attosecond generation and photoelectron emission in the streaking experiment drop significantly. Therefore, it would become even more important to lock the CEP at its optimum value to maximize the photon/photoelectron counts for the generation and characterization of 25 as XUV pulses, as well as for attosecond nonlinear experiments and any other attosecond experiments which require high photon flux.

\section{Conclusion}

In summary, the CE phase of the multi-pass and regenerative amplifier was both stabilized by changing the grating separation in stretcher or compressor. The grating-based CPA and CEphase control methods increased the energy of the CE phase stabilized laser pulse to the multi$\mathrm{mJ}$ level and the CE phase could be precisely controlled. The CE phase stabilization and control of these laser system are unambiguously confirmed by experimental observation of the $2 \pi$ periodicity of the high order harmonic spectrum generated by double optical gating. Therefore, CE-phase stable and controllable high-energy pulses are now a viable technology for studying ultrafast science. We have also demonstrated that the almost identical attosecond pulses can be generated at different CE phase values given the sufficient narrow gate width. However, the photon flux drops significantly if the CEP is tuned away from its optimum value for attosecond XUV pulse generation. This is true for both $9 \mathrm{fs}$ and $23 \mathrm{fs}$ lasers, where the $23 \mathrm{fs}$ NIR pulses were produced directly from a CPA amplifier. These studies pave the way for the realization of high-power $\mathrm{CE}$ phase stabilized lasers and high-flux single-isolated attosecond pulse generation, which are critical steps toward the study of nonlinear physics and pump probe experiments with single attosecond pulses.

Challenges do lie ahead for CE-phase-stabilization technology. For example, adaptive pulse shaping is a method where the phase of the laser pulse can be manipulated. If this method is combined with CE-phase stabilization and control, it could allow for the generation of ultrashort pulses with precise control of the absolute phase. Also, no group has actively stabilized and controlled the CE phase of even higher power laser system, such as TW class laser. This is also one of the major challenges future CE-phase research. Thus, there is room to improve in the area of CE-phase stabilization and control of Ti:sapphire laser amplifiers.

\section{References}

Baltuška, A., Udem, T., Uiberacker, M., Hentschel, M., Goulielmakis, E., Gohle, C., Holzwarth, R., Yakovlev, V. S., Scrinzi, A., Hänsch, T. W. \& Krausz, F. (2003). Attosecond control of electronic processes by intense light fields, Nature 421(6923): 611-615.

Barty, C. P. J., Guo, T., Blanc, C. L., Raksi, F., Rose-Petruck, C., Squier, J.,Wilson, K. R., Yakovlev, V. V. \& Yamakawa, K. (1996). Generation of 18-fs, multiterawatt pulses 
by regenerative pulse shaping and chirped-pulse amplification, Opt. Lett. 21(9): 668-670. URL: http://ol.osa.org/abstract.cfm?URI=ol-21-9-668

Chang, Z. (2006). Carrier-envelope phase shift caused by grating-based stretchers and compressors, Appl. Opt. 45(32): 8350-8353.

URL: http://ao.osa.org/abstract.cfm?URI=ao-45-32-8350

Chang, Z. (2007). Controlling attosecond pulse generation with a double optical gating, Phys. Rev. A 76(5): 051403.

Chen, S., Chini, M., Wang, H., Yun, C., Mashiko, H., Wu, Y. \& Chang, Z. (2009). Carrierenvelope phase stabilization and control of $1 \mathrm{khz}, 6 \mathrm{mj}, 30 \mathrm{fs}$ laser pulses from a ti:sapphire regenerative amplifier, Appl. Opt. 48(30): 5692-5695.

URL: http://ao.osa.org/abstract.cfm?URI=ao-48-30-5692

Chen, W.-J., Hsieh, Z.-M., Huang, S., Su, H.-Y., Lai, C.-J., Tang, T.-T., Lin, C.-H., Lee, C.-K., Pan, R.-P., Pan, C.-L. \& A. H. Kung, Phys. Rev. Lett. 100, . . (2008). Carrier-envelope phase-controlled quantum interference of injected photocurrents in semiconductors, Phys. Rev. Lett. 100: 163906.

Corkum, P. B. (1993). Plasma perspective on strong field multiphoton ionization, Phys. Rev. Lett. 71: 1993.

Corkum, P. B. \& Chang, Z. (2008). The attosecond revolution, Opt. Photonics News 19: 24.

Feng, X., Gilbertson, S., Mashiko, H., Wang, H., Khan, S. D., Chini, M., Wu, Y., Zhao, K. \& Chang, Z. (2009). Generation of isolated attosecond pulses with 20 to 28 femtosecond lasers, Phys. Rev. Lett. 103: 183901.

Fortier, T. M., Roos, P. A., Jones, D. J., Cundiff, S. T., Bhat, R. D. R. \& Sipe, J. E. (2004). Carrier-envelope phase-controlled quantum interference of injected photocurrents in semiconductors, Phys. Rev. Lett. 92: 147403.

Fuji, T., Rauschenberger, J., Apolonski, A., Yakovlev, V. S., Tempea, G., Udem, T., Gohle, C., Hänsch, T. W., Lehnert, W., Scherer, M. \& Krausz, F. (2005). Monolithic carrierenvelope phase-stabilization scheme, Opt. Lett. 30(3): 332-334.

URL: http:/ /ol.osa.org/abstract.cfm?URI=ol-30-3-332

Gilbertson, S., Khan, S. D., Wu, Y., Chini, M. \& Chang, Z. (2010). Isolated attosecond pulse generation without the need to stabilize the carrier-envelope phase of driving lasers, (Phys. Rev. Lett.) 105, 093902.

Gilbertson, S., Mashiko, H., Li, C., Moon, E. \& Chang, Z. (2008). Effects of laser pulse duration on extreme ultraviolet spectra from double optical gating, Applied Physics Letters 93(11): 111105. URL: http://link.aip.org/link/?APL/93/111105/1

Gilbertson, S., Wu, Y., Khan, S. D., Chini, M., Zhao, K., Feng, X. \& Chang, Z. (2010). Isolated attosecond pulse generation using multicycle pulses directly from a laser amplifier, Phys. Rev. A 81(4): 043810.

Haworth, C. A., Chipperfield, L. E., Robinson, J. S., Knight, P. L., Marangos, J. P. \& Tisch, J. W. G. (2007). Half-cycle cutoffs in harmonic spectra and robust carrier-envelope phase retrieval, Nature Phys. 3: 52-57.

Hentschel, M., Kienberger, R., Spielmann, C., Reider, G. A., Milosevic, N., Brabec, T., Corkum, P., Heinzmann, U., Drescher, M. \& Krausz, F. (2001). Attosecond metrology, Nature 414: 509.

Kakehata, M., Fujihira, Y., Takada, H., Kobayashi, Y., Torizuka, K., Homma, T. \& Takahashi, H. (2002). Measurements of carrier-envelope phase changes of 100-hz amplified laser pulses, Applied Physics B: Lasers and Optics 74(0): s43-s50.

URL: http:/ / dx.doi.org/10.1007/s00340-002-0880-1 
Kakehata, M., Takada, H., Kobayashi, Y., Torizuka, K., Fujihira, Y., Homma, T. \& Takahashi, H. (2001). Single-shot measurement of carrier-envelope phase changes by spectral interferometry, Opt. Lett. 26(18): 1436-1438.

URL: http:/ / ol.osa.org/abstract.cfm?URI=ol-26-18-1436

Kakehata, M., Takada, H., Kobayashi, Y., Torizuka, K., Takamiya, H., Nishijima, K., Homma, T., Takahashi, H., Okubo, K., Nakamura, S. \& Koyamada, Y. (2004). Carrier-envelopephase stabilized chirped-pulse amplification system scalable to higher pulse energies, Opt. Express 12(10): 2070-2080.

URL: http:// www.opticsexpress.org/abstract.cfm?URI=oe-12-10-2070

Kiriyama, H., Mori, M., Nakai, Y., Shimomura, T., Sasao, H., Tanoue, M., Kanazawa, S., Wakai, D., Sasao, F., Okada, H., Daito, I., Suzuki, M., Kondo, S., Kondo, K., Sugiyama, A., Bolton, P. R., Yokoyama, A., Daido, H., Kawanishi, S., Kimura, T. \& Tajima, T. (2010). High temporal and spatial quality petawatt-class Ti:sapphire chirped-pulse amplification laser system, OPTICS LETTERS 35(10): 1497-1499.

Kling, M. F., Siedschlag, C., Verhoef, A. J., Khan, J. I., Schultze, M., Uphues, T., Ni, Y., Uiberacker, M., Drescher, M., Krausz, F. \& Vrakking, M. J. J. (2006). Control of Electron Localization in Molecular Dissociation, Science 312(5771): 246-248.

URL: http://www.sciencemag.org/cgi/content/abstract/312/5771/246

Kreb, M., Löffler, T., Thomson, M. D., Dörner, R., Gimpel, H., Zrost, K., Ergler, T., Mashammer, R., Morgner, U., Ullrich, J. \& Roskos, H. G. (2006). Determination of the carrierenvelope phase of few-cycle laser pulses with terahertz-emission spectroscopy, Nature Phys. 2: 327-331.

Li, C., Mashiko, H., Wang, H., Moon, E., Gilbertson, S. \& Chang, Z. (2008). Carrier-envelope phase stabilization by controlling compressor grating separation, Applied Physics Letters 92(19): 191114. URL: http://link.aip.org/link/?APL/92/191114/1

Li, C., Moon, E. \& Chang, Z. (2006). Carrier-envelope phase shift caused by variation of grating separation, Opt. Lett. 31(21): 3113-3115.

URL: http:/ / ol.osa.org/abstract.cfm?URI=ol-31-21-3113

Li, C., Moon, E., Mashiko, H., Nakamura, C. M., Ranitovic, P., Maharjan, C. M., Cocke, C. L., Chang, Z. \& Paulus, G. G. (2006). Precision control of carrier-envelope phase in grating based chirped pulse amplifiers, Opt. Express 14(23): 11468-11476.

URL: http:/ / www.opticsexpress.org/abstract.cfm?URI=oe-14-23-11468

Mairesse, Y. \& Quéré, F. (2005). Frequency-resolved optical gating for complete reconstruction of attosecond bursts, Phys. Rev. A 71: 011401(R).

Mashiko, H., Gilbertson, S., Chini, M., Feng, X., Yun, C., Wanga, H., Khan, S. D., Chen, S. \& Chang, Z. (2009). Extreme ultraviolet supercontinua supporting pulse durations of less than one atomic unit of time, Opt. Lett. 34: 3337.

Mashiko, H., Gilbertson, S., Li, C., Khan, S. D., Shakya, M. M., Moon, E. \& Chang, Z. (2008). Double optical gating of high-order harmonic generation with carrierenvelope phase stabilized lasers, Phys. Rev. Lett. 100: 103906.

Mashiko, H., Nakamura, C. M., Li, C., Moon, E., Wang, H., Tackett, J. \& Changa, Z. (2007). Carrier-envelope phase stabilized 5.6 fs, 1.2 mj pulses, Appl. Phys. Lett. 90: 161114.

McKenna, J., Sayler, A. M., Anis, F., Gaire, B., Johnson, N. G., Parke, E., Hua, J. J., Mashiko, H., Nakamura, C. M., Moon, E., Chang, Z., Carnes, K. D., Esry, B. D. \& Ben-Itzhak, I. (2008). Enhancing high-order above-threshold dissociation of $h 2+$ beams with few-cycle laser pulses, Phys. Rev. Lett. 100(13): 133001. 
Moon, E., Li, C., Duan, Z., Tackett, J., Corwin, K. L., Washburn, B. R. \& Chang, Z. (2006). Reduction of fast carrier-envelope phase jitter in femtosecond laser amplifiers, Opt. Express 14(21): 9758-9763.

URL: http:/ / www.opticsexpress.org/abstract.cfm?URI=oe-14-21-9758

Moon, E., Wang, H., Gilbertson, S., Mashiko, H., Chini, M. \& Chang, Z. (2010). Advances in carrier-envelope phase stabilization of grating-based chirped-pulse amplifiers, Laser $\mathcal{E}$ Photonics Reviews 4(1): 160-177.

Paulus, G. G., Grasbon, F., Walther, H., Villoresi, P., Nisoli, M., Stagira, S., Priori, E. \& Silvestri, S. D. (2001). Absolute-phase phenomena in photoionization with fewcycle laser pulses, Nature 414: 182-184.

Sansone, G., Benedetti, E., Calegari, F., Vozzi, C., Avaldi, L., Flammini, R., Poletto, L., Villoresi, P., Altucci, C., Velotta, R., Stagira, S., Silvestri, S. D. \& Nisoli, M. (2006). Isolated single-cycle attosecond pulses, Science 314: 443.

Schultze, M., Fiess, M., Karpowicz, N., Gagnon, J., Korbman, M., Hofstetter, M., Neppl, S., Cavalieri, A. L., Komninos, Y., Mercouris, T., Nicolaides, C. A., Pazourek, R., Nagele, S., Feist, J., Burgdorfer, J., Azzeer, A. M., Ernstorfer, R., Kienberger, R., Kleineberg, U., Goulielmakis, E., Krausz, F. \& Yakovlev, V. S. (2010). Delay in Photoemission, Science 328(5986): 1658-1662.

URL: http://www.sciencemag.org/cgi/content/abstract/328/5986/1658

Shan, B. \& Chang, Z. (2001). Dramatic extension of the high-order harmonic cutoff by using a long-wavelength driving field, Phys. Rev. A 65(1): 011804.

Sola, I. J., Mével, E., Elouga, L., Constant, E., Strelkov, V., Poletto, L., Villoresi, P., Benedetti, E., Caumes, J.-P., Stagira, S., Vozzi, C., Sansone, G. \& Nisoli, M. (2006). Controlling attosecond electron dynamics by phase-stabilized polarization gating, Nat. Phys. 2: 319.

Strickland, D. \& Mourou, G. (1985). Compression of amplified chirped optical pulses, Optics Communications 55(6): $447-449$.

URL: http:/ / www.sciencedirect.com/science/article/B6TVF-46JGW3M1BX/2/ef583949f08c6e26c541eeff02c32fa1

Thomann, I., Gagnon, E., Jones, R., Sandhu, A., Lytle, A., Anderson, R., Ye, J., Murnane, M. \& Kapteyn, H. (2004). Investigation of a grating-based stretcher/compressor for carrier-envelope phase stabilized fs pulses, Opt. Express 12(15): 3493-3499.

URL: http://www.opticsexpress.org/abstract.cfm?URI=oe-12-15-3493

Tzallas, P., Charalambidis, D., Papadogiannis, N. A., Witte, K. \& Tsakiris, G. D. (2003). Direct observation of attosecond light bunching, Nature 426(6964): 267-271.

URL: http://dx.doi.org/10.1038/nature02091

Vaillancourt, G., Norris, T. B., Coe, J. S., Bado, P. \& Mourou, G. A. (1990). Operation of a 1khz pulse-pumped ti:sapphire regenerative amplifier, Opt. Lett. 15(6): 317-319.

URL: http:/ / ol.osa.org/abstract.cfm?URI=ol-15-6-317

Wang, H., Chini, M., Khan, S. D., Chen, S., Gilbertson, S., Feng, X., Mashiko, H. \& Chang, Z. (2009). Practical issues of retrieving isolated attosecond pulses, Journal of Physics B: Atomic, Molecular and Optical Physics 42(13): 134007.

URL: http:/ / stacks.iop.org/0953-4075/42/i=13/a=134007

Yun, C., Chen, S., Wang, H., Chini, M. \& Chang, Z. (2009). Temperature feedback control for long-term carrier-envelope phase locking, Appl. Opt. 48(27): 5127-5130.

URL: http://ao.osa.org/abstract.cfm?URI=ao-48-27-5127 


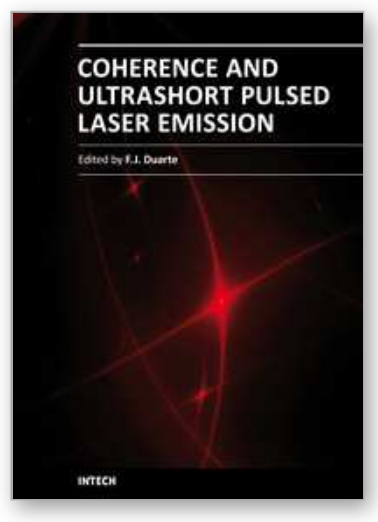

\section{Coherence and Ultrashort Pulse Laser Emission}

Edited by Dr. F. J. Duarte

ISBN 978-953-307-242-5

Hard cover, 688 pages

Publisher InTech

Published online 30, November, 2010

Published in print edition November, 2010

In this volume, recent contributions on coherence provide a useful perspective on the diversity of various coherent sources of emission and coherent related phenomena of current interest. These papers provide a preamble for a larger collection of contributions on ultrashort pulse laser generation and ultrashort pulse laser phenomena. Papers on ultrashort pulse phenomena include works on few cycle pulses, high-power generation, propagation in various media, to various applications of current interest. Undoubtedly, Coherence and Ultrashort Pulse Emission offers a rich and practical perspective on this rapidly evolving field.

\section{How to reference}

In order to correctly reference this scholarly work, feel free to copy and paste the following:

Shouyuan Chen, Yi Wu, Kun Zhao and Zenghu Chang (2010). Carrier-Envelope Phase Stablization of Grating Based High-Power Ultrafast Laser, Coherence and Ultrashort Pulse Laser Emission, Dr. F. J. Duarte (Ed.), ISBN: 978-953-307-242-5, InTech, Available from: http://www.intechopen.com/books/coherence-andultrashort-pulse-laser-emission/carrier-envelope-phase-stablization-of-grating-based-high-power-ultrafastlaser-

\section{INTECH}

open science | open minds

\section{InTech Europe}

University Campus STeP Ri Slavka Krautzeka 83/A 51000 Rijeka, Croatia Phone: +385 (51) 770447 Fax: +385 (51) 686166 www.intechopen.com

\section{InTech China}

Unit 405, Office Block, Hotel Equatorial Shanghai No.65, Yan An Road (West), Shanghai, 200040, China 中国上海市延安西路65号上海国际贵都大饭店办公楼 405 单元 Phone: +86-21-62489820

Fax: $+86-21-62489821$ 
(C) 2010 The Author(s). Licensee IntechOpen. This chapter is distributed under the terms of the Creative Commons Attribution-NonCommercialShareAlike-3.0 License, which permits use, distribution and reproduction for non-commercial purposes, provided the original is properly cited and derivative works building on this content are distributed under the same license. 